\title{
Postoperative sore throat: inevitable side effect or preventable nuisance?
}

\author{
Alana M. Flexman, MD, FRCPC (1) - Laura V. Duggan, MD, FRCPC
}

Received: 20 March 2019/Revised: 20 March 2019/ Accepted: 13 May 2019/Published online: 21 May 2019

(C) Canadian Anesthesiologists' Society 2019

In this issue of the Journal, Kuriyama et al. ${ }^{1}$ present a well-conducted systematic review and meta-analysis showing that topical magnesium is highly effective in preventing postoperative sore throat (POST) after endotracheal intubation. Although POST is often considered a minor complication of anesthesia, it remains one of the most common patient complaints after general anesthesia with an incidence of $20-40 \%$. $^{2,3}$ Furthermore, anesthesiologists may not be aware of the incidence of sore throat in their own practice, as many patients may not seek medical advice for POST. In a large $(n=12,276)$ prospective cohort study of a broad cross section of surgical patients, $20 \%$ of patients complained of sore throat after general anesthesia, second only to postoperative nausea and vomiting. ${ }^{2}$ Female gender and younger age were identified as risk factors. Another large $(n=809)$ prospective study identified a higher incidence of $40 \%$ as well as identifying additional independent risk factors, including a history of smoking or lung disease, longer duration of anesthesia, postoperative nausea, natural teeth ( $v s$ dentures), and blood visible on the endotracheal tube at extubation. ${ }^{3}$ Still, other risk factors for POST have been identified including various intubation techniques ${ }^{4}$ and the use of larger diameter double lumen endotracheal tubes. ${ }^{5}$ Fortunately, POST

\footnotetext{
A. M. Flexman, MD, FRCPC $(\bowtie) \cdot$ L. V. Duggan, MD, FRCPC Department of Anesthesiology and Perioperative Care, Vancouver General Hospital, 899 West 12th Avenue, Vancouver, BC V5Z 1M9, Canada

e-mail: alana.flexman@vch.ca
}

Department of Anesthesiology, Pharmacology and Therapeutics, University of British Columbia, Vancouver, BC, Canada appears short-lived with most cases resolving within $48 \mathrm{hr}$ of anesthesia. ${ }^{3}$

Although anesthesiologists may consider this complication "minor," patients who experience a sore throat after their surgery may not agree. As Kuriyama et al. note, the presence of a sore throat after anesthesia can contribute to patients' dissatisfaction with anesthesia ${ }^{6}$ and interfere with their return to function, including eating and drinking. Fortunately, several effective strategies to prevent and/or treat sore throat after general anesthesia have been described in the literature, including intraoperative techniques to reduce the incidence and severity. These same authors have recently published several other systematic reviews and meta-analyses summarizing the literature describing other effective treatments of POST, including intravenous dexamethasone (pooled risk ratio [RR], 0.62; 95\% confidence interval [CI], 0.51 to 0.75 ), ${ }^{7}$ topical benzydamine (RR, $0.31 ; 95 \% \mathrm{CI}, 0.20$ to $0.47),{ }^{8}$ and topical corticosteroids (RR, 0.39; $95 \%$ CI, 0.32 to 0.49). ${ }^{9}$ Multiple other treatments have been studied and found to be effective at reducing the incidence and severity of sore throat including lidocaine (either intra-cuff ${ }^{10}$ or intravenously administered), ${ }^{11}$ ketamine, ${ }^{12}$ with even the choice of volatile anesthetic playing a role (desflurane is associated with a higher incidence of POST than sevoflurane). ${ }^{13}$

The current systematic review and meta-analysis by Kuriyama et al. provides an opportunity to consolidate the literature supporting topical magnesium as a highly effective treatment for POST. The authors examined both published and unpublished studies, and their search strategy was robust. In total, the authors found eleven studies matching their search criteria. The population was adults greater than $18 \mathrm{yr}$ old (of either sex) undergoing elective general anesthesia and tracheal intubation for a wide range of elective non-cardiac procedures, enhancing the generalizability of their findings. All studies used a one-time dose of magnesium prior to 
tracheal intubation. Both dose $\left(100-500 \mathrm{mg}, 20 \mathrm{mg} \cdot \mathrm{kg}^{-1}\right.$, or not stated at all) and delivery (gargle, lozenge, or nebulization) of topical magnesium were highly variable. Depending on the specific study, topical magnesium was compared with either a non-analgesic control or an analgesic control with known benefit for POST. Presence and severity of POST were the primary and secondary outcomes, respectively, and POST severity was measured using a multitude of visual analogue scales (e.g., 0-5, 0-10, 0-100) or an ordinal scale (none, mild, moderate, or severe POST).

In the eight studies that provided data of POST $24 \mathrm{hr}$ after surgery, the pooled results showed a clinically important decrease in the incidence of POST with the application of topical magnesium (RR, 0.31; 95\% CI, 0.21 to 0.45 ). Similarly, when POST occurred, its severity was reduced when magnesium was used (standardized mean difference, $-2.7 ; 95 \% \mathrm{CI},-3.9$ to -1.4$)$. Compared with a non-analgesic control, the use of topical magnesium was associated with a decrease in voice hoarseness. Interestingly, a further sensitivity analysis suggested that nebulized magnesium was more effective than gargling. In general, this treatment was well-tolerated; there were no adverse effects associated with the use of topical magnesium identified in any of the studies.

The results are compelling, but the study has limitations. Although the authors attempted to perform a meta-regression to identify a dose-response effect, Kuriyama et al. were unable to provide information on the optimal dose or the specific mode of delivery (nebulized, gargle, or lozenge). In fact, the doses provided in the studies were heterogeneous and not always weight-based. All included studies were performed outside North America, therefore whether these findings are generalizable to the patient population and practice in Canada remains unclear. In addition, magnesium has not been shown to be superior to other analgesic interventions described above, only to non-analgesic alternatives. Although something may indeed be better than nothing, the most efficacious intervention to prevent POST remains unknown.

Despite this robust, well-conducted systematic review and meta-analysis showing a highly effective treatment for a common complication of anesthesia, this therapeutic intervention remains under-used in Canada. A large part of the problem is that no commercially available topical magnesium preparation is available in Canada for this purpose, which clearly presents a barrier to its use in the operating room. In addition, anesthesiologists in Canada may not be aware of the efficacy of topical magnesium in preventing POST. In preparation for this present editorial, the authors conducted an informal Twitter poll ${ }^{14}$ to assess the level of awareness amongst our followers. Out of the 285 votes, only $1 \%$ of respondents agreed that topical

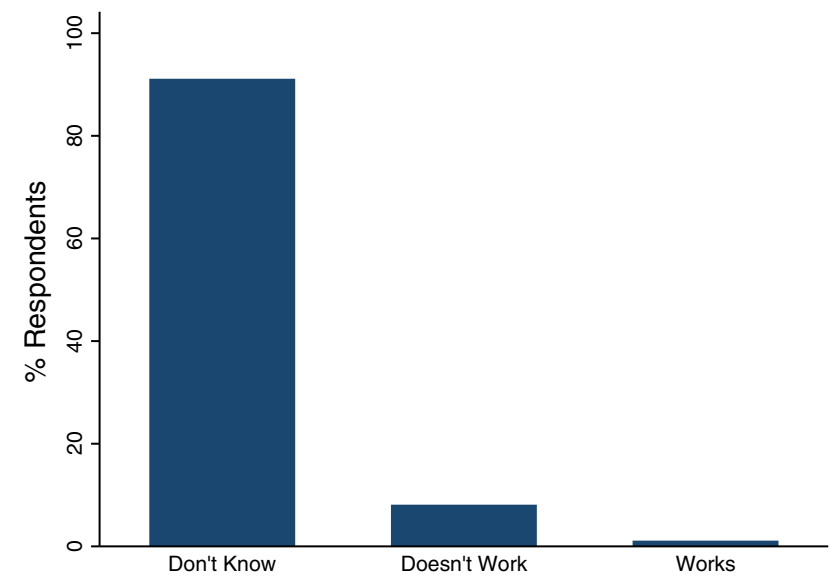

Figure Results of an informal poll $(n=285)$ on Twitter about the efficacy of topical magnesium on postoperative sore throat. Available from URL: https://twitter.com/drlauraduggan/status/ 1098378238550654976Résultats d'un sondage informel $(\mathrm{n}=285)$ sur la plateforme Twitter concernant l'efficacité du magnésium en application topique pour prévenir les maux de gorge postopératoires. Disponible en suivant le lien: https://twitter.com/drlauraduggan/ status/1098378238550654976

magnesium is effective for POST (Figure). These results confirmed our suspicions that magnesium is an underappreciated therapy, despite its effectiveness, and they highlight the need for further knowledge translation. As anesthesiologists, we should take this opportunity to advocate for a formulation amenable to this application, either at the local or national level.

Ultimately, where does this leave us in Canada? As Canadian healthcare continues to move toward embracing patient-centred health outcomes, POST may begin to be viewed as a preventable side effect versus an inevitable "nuisance" outcome of tracheal intubation. With the current focus on early enhanced recovery from anesthesia and surgery (ERAS), it is not unreasonable to view prevention of POST as part of any ERAS patient care initiative. Patients wish to return to full function as early as possible, including eating and drinking, and we as anesthesiologists should be part of the solution.

\section{Maux de gorge postopératoires : effet secondaire inéluctable ou désagrément évitable?}

Dans ce numéro du Journal, Kuriyama et coll. présentent une revue systématique et une méta-analyse bien réalisées et démontrant que le magnésium en application topique est 
très efficace pour prévenir les maux de gorge postopératoires suivant l'intubation endotrachéale. Bien que les maux de gorge postopératoires soient souvent considérés comme une complication mineure de l'anesthésie, ils demeurent l'une des plaintes les plus fréquentes après une anesthésie générale, l'incidence étant de l'ordre de 20-40\%. ${ }^{2,3}$ En outre, les anesthésiologistes pourraient ne pas être conscients de l'incidence des maux de gorge dans leur propre pratique, étant donné que bon nombre de patients ne consulteront vraisemblablement pas leur médecin pour des maux de gorge postopératoires. Dans une importante étude de cohorte prospective $(n=12$ 276) englobant une vaste section transversale de patients chirurgicaux, $20 \%$ des patients se sont plaints de maux de gorge après l'anesthésie générale, ce qui en fait le deuxième effet secondaire le plus fréquemment rapporté, juste après les nausées et vomissements postopératoires. ${ }^{2}$ Le sexe féminin et un jeune âge ont été identifiés comme facteurs de risque. Une autre importante étude prospective ( $n=809)$ a rapporté une incidence plus élevée atteignant $40 \%$ ainsi que d'autres facteurs de risque indépendants supplémentaires, notamment des antécédents de tabagisme ou de maladie pulmonaire, une durée prolongée de l'anesthésie, des nausées postopératoires, des dents naturelles ( $v s$ un dentier), et la présence de sang visible sur le tube endotrachéal lors de l'extubation. ${ }^{3}$ D'autres facteurs de risque de maux de gorge postopératoires ont également été identifiés, y compris diverses techniques d'intubation ${ }^{4}$ et l'utilisation de tubes endotrachéaux à double lumière de diamètres plus gros. ${ }^{5}$ Heureusement, les maux de gorge postopératoires semblent être de courte durée, la plupart des cas se résolvant dans les 48 h suivant l'anesthésie. ${ }^{3}$

Même si les anesthésiologistes peuvent considérer cette complication comme étant «mineure », les patients souffrant de maux de gorge après leur chirurgie pourraient ne pas être d'accord. Comme le notent Kuriyama et coll., la présence de maux de gorge après l'anesthésie peut contribuer à l'insatisfaction des patients concernant leur anesthésie ${ }^{6}$ et entraver leur rétablissement fonctionnel, notamment l'ingestion de nourriture et de liquides. Plusieurs stratégies efficaces ont heureusement été décrites dans la littérature pour prévenir et/ou traiter les maux de gorge subséquents à une anesthésie générale, notamment des techniques peropératoires visant à en réduire l'incidence et la gravité. Ces mêmes auteurs ont récemment publié plusieurs autres revues systématiques et méta-analyses résumant la littérature décrivant d'autres traitements efficaces pour les maux de gorge postopératoires, y compris la dexaméthasone intraveineuse (risque relatif [RR] combiné, 0,62; intervalle de confiance [IC] $95 \%, 0,51$ à 0,75$){ }^{7}$ la benzydamine topique (RR, 0,31; IC $95 \%, 0,20$ à 0,47$),{ }^{8}$ et les corticostéroïdes topiques (RR, 0,39; IC 95\%, 0,32 à 0,49). ${ }^{9}$ Plusieurs autres traitements ont été étudiés et leur efficacité a été démontrée pour réduire l'incidence et la gravité des maux de gorge, comme par exemple la lidocaïne (à l'intérieur du ballonnet ${ }^{10}$ ou par administration intraveineuse), ${ }^{11}$ la kétamine, ${ }^{12}$ le choix d'agent anesthésique volatile jouant lui-même un rôle (par exemple, le desflurane est associé à une incidence plus élevée de maux de gorge postopératoires que le sévoflurane). ${ }^{13}$

La revue systématique et la méta-analyse de Kuriyama et coll. rapportées dans ce numéro du Journal nous offrent l'occasion de consolider la littérature en faveur de l'utilisation topique de magnésium en tant que traitement très efficace des maux de gorge postopératoires. Les auteurs ont passé en revue les études publiées et non publiées, et leur stratégie de recherche fut rigoureuse. Au total, les auteurs ont examiné onze études respectant leurs critères de recherche. La population était constituée d'adultes de plus de 18 ans (hommes et femmes) subissant une anesthésie générale et une intubation trachéale non urgentes pour toutes sortes d'interventions non cardiaques non urgentes, ce qui améliore l'aspect généralisable de leurs résultats. Toutes les études ont utilisé une dose unique de magnésium avant l'intubation trachéale. Les doses (100-500 $\mathrm{mg}, 20 \mathrm{mg} \cdot \mathrm{kg}^{-1}$, ou inconnue) et les méthodes d'administration (gargarisme, bonbon ou nébulisation) du magnésium topique étaient très variables. Selon les études, le magnésium topique a été comparé soit à un témoin non analgésique, soit à un traitement analgésique ayant des bienfaits connus pour prévenir les maux de gorge postopératoires. La présence et la gravité des maux de gorge postopératoires constituaient les critères d'évaluation principal et secondaire, respectivement, et la gravité des maux de gorge postopératoires a été mesurée à l'aide d'une multitude d'échelles visuelles analogiques (par ex., 0-5, 0-10, 0-100) ou d'une échelle ordinale (aucun mal de gorge, maux de gorge postopératoires légers, modérés ou sévères).

Dans les huit études présentant des données sur les maux de gorge postopératoires $24 \mathrm{~h}$ après la chirurgie, les résultats combinés montraient une réduction cliniquement importante de l'incidence des maux de gorge postopératoires lors de l'application topique de magnésium (RR, 0,31; IC $95 \%, 0,21$ à 0,45). De la même manière, lorsque des maux de gorge postopératoires sont apparus, leur gravité était réduite lors de l'utilisation de magnésium (différence moyenne standardisée, -2,7; IC $95 \%,-3,9$ à -1,4). Par rapport à une intervention témoin non analgésique, l'utilisation topique de magnésium a été associée à une réduction de l'enrouement de la voix. Fait intéressant, une analyse de sensibilité approfondie a suggéré que le magnésium nébulisé était plus efficace 
que le gargarisme. Le traitement a été bien toléré en général; aucun effet indésirable associé à l'utilisation topique de magnésium n'a été identifié dans ces études.

Ces résultats sont convaincants, mais l'étude comporte certaines limites. Bien que les auteurs aient tenté de réaliser une méta-régression afin d'identifier un effet de dose-réponse, Kuriyama et coll. n'ont pas pu fournir d'informations quant à la dose ou au mode d'administration spécifique (par nébulisation, gargarisme ou bonbon) optimaux. En fait, les doses administrées dans les études étaient très hétérogènes et ne se basaient pas systématiquement sur le poids. Toutes les études incluses ont été réalisées hors Amérique du Nord; par conséquent, nous ne savons pas si ces résultats peuvent être appliqués à la population de patients et à la pratique canadiennes. En outre, il n'a pas été démontré que le magnésium était supérieur aux autres interventions analgésiques énumérées ci-dessus - seulement aux alternatives non analgésiques. Bien que toute intervention efficace soit utile, nous ne savons toujours pas laquelle de ces interventions est la plus efficace pour prévenir les maux de gorge postopératoires.

Malgré cette revue systématique et cette méta-analyse rigoureuses et bien réalisées, qui présentent un traitement très efficace pour une complication courante de l'anesthésie, cette intervention thérapeutique demeure sous-utilisée au Canada. Une grande partie du problème réside dans le fait qu'il n'existe pas de préparation commerciale de magnésium topique facilement disponible au Canada pour cette application, ce qui représente clairement un obstacle à son utilisation en salle d'opération. En outre, les anesthésiologistes au Canada pourraient ne pas bien connaître l'efficacité du magnésium topique pour la prévention des maux de gorge postopératoires. Pour préparer cet éditorial, les auteurs ont réalisé un sondage informel sur Twitter ${ }^{14}$ afin d'évaluer le niveau de connaissances de cet agent parmi nos abonnés. Sur les 285 votes, seul $1 \%$ des répondants étaient d'accord que le magnésium topique est efficace pour prévenir les maux de gorge postopératoires (voir figure). Ces résultats ont confirmé nos soupçons : le magnésium constitue un traitement peu apprécié malgré son efficacité; un transfert plus important des connaissances est nécessaire. En tant qu'anesthésiologistes, nous devrions saisir cette occasion d'exiger une formule qui se prête à cette application, tant au niveau local que national.

Où cela nous laisse-t-il au Canada en bout de ligne? Alors que le système de santé canadien continue de progresser vers des pronostics de santé centrés sur le patient, les maux de gorge postopératoires pourraient commencer à être perçus comme un effet secondaire évitable plutôt qu'un «désagrément» de l'intubation trachéale auquel on ne peut échapper. Étant donné l'engouement actuel pour la récupération rapide améliorée après l'anesthésie et la chirurgie (ERAS), il semble raisonnable de prendre en compte la prévention des maux de gorge postopératoires dans le cadre de toute initiative de soins destinés aux patients en ERAS. Les patients souhaitent récupérer toutes leurs fonctions vitales le plus tôt possible, y compris l'ingestion de nourriture et de liquides et, en tant qu'anesthésiologistes, nous nous devons de faire partie de la solution.

Disclosures and conflicts of interest Dr. Alana M. Flexman and Dr. Laura V. Duggan have no disclosures or conflicts of interest in connection to this manuscript.

Editorial responsibility This submission was handled by Dr. Philip M. Jones, Associate Editor, Canadian Journal of Anesthesia.

Déclarations et conflit d'intérêt Dre Alana M. Flexman et Dre Laura V. Duggan n'ont aucune déclaration ou conflit d'intérêt à déclarer par rapport à ce manuscrit.

Responsabilité Éditoriale Cet article a été traité par Dr Philip M. Jones, rédacteur adjoint, Journal canadien d'anesthésie.

\section{References}

1. Kuriyama A, Maeda H, Sun R. Topical application of magnesium to prevent intubation-related sore throat in adult surgical patients: a systematic review and meta-analysis. Can J Anesth 2019; 66: DOI: https://doi.org/10.1007/s12630-019-01396-7.

2. Lehmann $M$, Monte $K$, Barach $P$, Kindler $\mathrm{CH}$. Postoperative patient complaints: a prospective interview study of 12,276 patients. J Clin Anesth 2010; 22: 13-21.

3. Biro P, Seifert B, Pasch T. Complaints of sore throat after tracheal intubation: a prospective evaluation. Eur J Anaesthesiol 2005; 22: 307-11.

4. El-Boghdadly $K$, Bailey $C R$, Wiles $M D$. Postoperative sore throat: a systematic review. Anaesthesia 2016; 71: 706-17.

5. Clayton-Smith A, Bennett $K$, Alston RP, et al. A comparison of the efficacy and adverse effects of double-lumen endobronchial tubes and bronchial blockers in thoracic surgery: a systematic review and meta-analysis of randomized controlled trials. J Cardiothorac Vasc Anesth 2015; 29: 955-66.

6. Tong D, Chung F, Wong D. Predictive factors in global and anesthesia satisfaction in ambulatory surgical patients. Anesthesiology 1997; 87: 856-64.

7. Kuriyama A, Maeda $H$. Preoperative intravenous dexamethasone prevents tracheal intubation-related sore throat in adult surgical patients: a systematic review and meta-analysis. Can J Anesth 2019; DOI: https://doi.org/10.1007/s12630-018-01288-2.

8. Kuriyama A, Aga $M$, Maeda $H$. Topical benzydamine hydrochloride for prevention of postoperative sore throat in adults undergoing tracheal intubation for elective surgery: a systematic review and meta-analysis. Anaesthesia 2018; 73: 889900.

9. Kuriyama A, Maeda $H$, Sun R, Aga M. Topical application of corticosteroids to tracheal tubes to prevent postoperative sore throat in adults undergoing tracheal intubation: a systematic review and meta-analysis. Anaesthesia 2018; 73: 1546-56.

10. Lam F, Lin YC, Tsai HC, Chen TL, Tam KW, Chen $C Y$. Effect of intracuff lidocaine on postoperative sore throat and the 
emergence phenomenon: a systematic review and meta-analysis of randomized controlled trials. PLoS One 2015; 10: e0136184.

11. Tanaka Y, Nakayama T, Nishimori M, Tsujimura Y, Kawaguchi $M$, Sato $Y$. Lidocaine for preventing postoperative sore throat. Cochrane Database Syst Rev 2015; 7: CD004081.

12. Mayhood J, Cress K. Effectiveness of ketamine gargle in reducing postoperative sore throat in patients undergoing airway instrumentation: a systematic review. JBI Database System Rev Implement Rep 2015; 13: 244-78.

13. Park JH, Lee YC, Lee J, Kim S, Kim HC. Influence of intraoperative sevoflurane or desflurane on postoperative sore throat: a prospective randomized study. J Anesth 2019; DOI: https://doi.org/10.1007/s00540-018-2600-y.

14. Twitter Poll. What is your opinion about the utility of topical magnesium in treating post-operative sore throat? Available from URL: https://twitter.com/drlauraduggan/status/ 1098378238550654976 (accessed March 2019).

Publisher's Note Springer Nature remains neutral with regard to jurisdictional claims in published maps and institutional affiliations. 